\title{
Azathioprine as a Neuroprotective Agent in Experimental Traumatic Spinal Cord Injury
}

\author{
Sinan BAHADIR ${ }^{1}$, Mehmet Bulent ONAL ${ }^{2}$, Firat NARIN ${ }^{3}$, Ibrahim BASAR ${ }^{4}$, Kazim YIGITKANLI $^{5}$, Murad BAVBEK ${ }^{6}$, \\ Mustafa Ibrahim ZIYAL ${ }^{7}$
}

\author{
${ }^{1}$ Amasya University Medicine Faculty, Department of Neurosurgery, Amasya, Turkey \\ ${ }^{2}$ Acıbadem Mehmet Ali Aydınlar University, Vocational School of Health Sciences, Istanbul, Turkey \\ ${ }^{3}$ Van Yuzuncuyil Training and Research Hospital, Neurosurgery Clinic, Van, Turkey \\ ${ }^{4}$ Dicle University School of Medicine, Department of Neurosurgery, Diyarbakir, Turkey \\ ${ }^{5}$ Medicana Bursa Hospital, Neurosurgery Clinic, Bursa, Turkey \\ ${ }^{6}$ Ankara Yıldırım Beyazıt University, Department of Neurosurgery, Ankara, Turkey \\ ${ }^{7}$ Marmara University, Department of Neurosurgery, Istanbul, Turkey
}

Corresponding author: Sinan BAHADIR sinanbahadir@windowslive.com

\section{ABSTRACT}

AIM: To evaluate the protective effects of azathioprine, a macrophage-inhibiting agent, on secondary injury in spinal cord trauma.

MATERIAL and METHODS: A total of 40 Wistar rats were randomly divided into 4 groups. All the animals had undergone T8-10 laminectomy. Except in group I (control), all the animals were exposed to spinal cord trauma at the T9 level. Animals in group II (trauma) received no treatment following trauma. Animals in group 3 (treatment) and group IV (vehicle) were given intraperitoneal azathioprine $4 \mathrm{mg} / \mathrm{kg}$ and saline $2 \mathrm{ml}$, respectively, 30 minutes after the trauma. Half of the animals in each group were sacrificed 24 hours after injury and specimens were used for biochemical and immunohistochemical evaluations. The rest of the animals were followed-up for 4 weeks in terms of neurological functions and were also sacrificed to perform the histopathological analysis.

RESULTS: Significant decrease in apoptotic cells and improved neurological function were observed in the animals treated with azathioprine. Biological and immunohistochemical analysis also showed less oxidative stress in this group compared to those without treatment.

CONCLUSION: Azathioprine, a potent macrophage-inhibiting agent, has been shown to decrease the extent of secondary injury following spinal cord trauma.

KEYWORDS: Trauma, Spinal cord injury, Secondary injury, Azathioprine, Rat

ABBREVIATIONS: APAF-1: Apoptotic peptidase activating factor 1, DNA: Deoxyribonucleic acid, eNOS: Endothelial nitric oxide synthase, iNOS: Inducible nitric oxide synthase, IPS: Inclined plane score, MDA: Malondialdehyde, MFS: Motor function score, NOS: Nitrix oxide synthase, PC: Protein carbonyl, rGSH: Reduced glutathione, SCI: Spinal cord injury, SD: Standard deviation, TNF-a: Tumor necrosis factor a

\section{INTRODUCTION}

A cute traumatic spinal cord injury ( $\mathrm{SCl}$ ) is a serious problem that diversely affects not only the individual, but also their family and the whole society diversely.
Following trauma, the initial primary mechanical injury is followed by progressive secondary injury events involving ischemia, edema, excitotoxicity, and inflammation $(2,13,23)$. Generally, it is the secondary injury that defines the outcome,
Sinan BAHADIR (1D): 0000-0002-1037-5645

Mehmet Bulent ONAL (1) : 0000-0003-0563-3221

Firat NARIN (10): 0000-0002-5985-4460
Ibrahim BASAR (1) : 0000-0003-3674-4864

Kazim YIGITKANLI (1) : 0000-0003-0628-0173

Murad BAVBEK (1): 0000-0002-6256-4202
Mustafa Ibrahim ZIYAL (1) : 0000-0001-6142-1085 
since the amount of spared neural tissue is directly related to the functional recovery (22). In the early stages of injury, proinflammatory mediators such as cytokines and chemokines are released rapidly and in response, macrophages and neutrophils infiltrate the tissue, thus becoming major contributors in the later stages of the inflammatory process $(4,22)$.

Azathioprine is a purine antimetabolite that inhibits protein synthesis and cell proliferation through the inhibition of deoxyribonucleic acid (DNA) synthesis. It is used for its immunosuppressive effects after organ transplantations and anti-inflammatory effects in inflammatory bowel diseases. It is slowly metabolized into 6-mercaptopurine after administration. Although it affects mainly $\mathrm{T}$ cells, several important effects on macrophages have also been documented (5). It has been shown to decrease macrophage infiltration into the peritoneal cavity following inflammation (9). Additionally, it was shown to inhibit inducible nitric oxide synthase (iNOS) production in macrophages (18). Furthermore, azathioprine has neuroprotective effects by decreasing the apoptosis following spinal cord ischemia-reperfusion injury (1). To the best of our knowledge, the effect of azathioprine has not yet been studied in a spinal cord trauma model.

We hypothesize that due to its immunosuppressive and anti-inflammatory effects, azathioprine will decrease the effects of secondary injury in a spinal cord trauma setting. In this experimental study, we aimed to investigate the neuroprotective, neurobehavioral, and anti-apoptotic effects of azathioprine in an experimental spinal cord trauma model.

\section{MATERIAL and METHODS}

A total of forty 6-8-week-old male Wistar rats weighing 120$150 \mathrm{~g}$ were used in this study. Approval for our study was obtained from the Hacettepe University Animal Research Committee (Date: 06.06.2006, Decision number: 2006/48-7). Animals were housed in separate cages and had ad libitum access to food and water throughout the study.

The rats were assigned to the following groups, with each group comprising of 10 rats. Rats in group 1 (Control group) underwent laminectomy, but were not exposed to trauma. Rats in group 2 (Trauma group) were exposed to spinal cord trauma but did not receive any medication. Rats in group 3 (Treatment group) were given azathioprine (Imuran, Glaxo Smith Kline, Istanbul, Turkey) $4 \mathrm{mg} / \mathrm{kg}$ (2 $\mathrm{mL}$, dissolved in saline) intraperitoneally, 30 minutes after the surgery and via oral gavage ( $2 \mathrm{~mL}$, dissolved in saline) at 12, 24, 36, and 48 hours after the procedure. Rats in group 4 (Vehicle group) received $2 \mathrm{~mL}$ of standard saline solution intraperitoneally, 30 min after the injury and via oral gavage at 12, 24, 36, and 48 hours after the injury.

Later, half of the animals in each group were sacrificed 24 hours after the surgery and spinal cord samples were collected for biochemical and immunohistochemical evaluations. The remaining rats were followed-up for 4 weeks, in order to perform neurobehavioral assessments. These rats were also sacrificed at the end of the observation period and spinal cord samples were collected to evaluate the histopathological changes.

\section{Injury Procedure}

Anesthesia was performed by an intraperitoneal injection of ketamine $60 \mathrm{mg} / \mathrm{kg}$ (Ketalar $5 \%$ solution, Eczacıbasi llac Sanayi under Parke-Davis license, Istanbul, Turkey) and xylazine 8 mg/kg (Rompun 2\% solution, Bayer, Istanbul, Turkey). Following a dorsal midline incision approximately between the T5-T12 levels, the paravertebral muscles were dissected and the thoracic vertebrae from T7-T11 were exposed. Then, T8-10 laminectomies were performed under microscopy to expose the spinal cord, but the dura was left intact. Spinal cord injury was induced using a weight-dropping technique that was modified from Allen's method (12). The animals were exposed to an impact of $50 \mathrm{~g} / \mathrm{cm}$ on the dorsal surface of the spinal cord by dropping a $5 \mathrm{~g}$ weight of lead through a $10-\mathrm{cm}$ guide tube that was positioned perpendicular to the center of spinal cord. Afterwards, the muscles and skin were sutured and the animals recovered in temperature and humiditycontrolled chambers.

\section{Biochemical Examination}

To assess oxidative damage, we evaluated the lipid peroxidation, protein carbonyl, and glutathione peroxidase levels.

Lipid peroxidation was assessed by measuring the thiobarbituric acid-malondialdehyde (MDA) complexes. Results were obtained as nanomole per gram of wet tissue $(\mathrm{nmol} / \mathrm{g})$.

Protein carbonyl (PC) levels were also measured to determine oxidative protein damage. Results were expressed as nanomole per milligram of tissue $(\mathrm{nmol} / \mathrm{mg})$.

Reduced glutathione ( $\mathrm{rGSH}$ ) contents of the tissues were assessed using assay kits from Cayman Chemical Company (Ann Arbor. MI, USA). Results were expressed as nanomole per gram of tissue (nmol/g).

Detailed descriptions of the measurement techniques for these markers can be found elsewhere $(7,15,24)$.

\section{Immunohistochemical Examination}

For immunohistochemical evaluation, paraffin-embedded blocks were cut into $5 \mu \mathrm{m}$ sections and applied to xylene, washed in ethanol and water, treated with $3 \%$ hydrogen peroxide, and washed with phosphate-buffered saline. Sections incubated at $4^{\circ} \mathrm{C}$ with an iNOS rabbit polyclonal antibody (Lab Vision Corp., Fremont, CA, USA), endothelial NOS (eNOS) rabbit polyclonal antibody (Lab Vision Corp., Fremont, CA, USA), and apoptotic peptidase-activating factor 1 (APAF-1) epitope-specific rabbit antibody (Lab Vision Corp., Fremont, CA, USA).

Slides were examined under light microscope by a blinded histopathologist. For each animal, three sections around the lesion epicenter were examined and five regions per section were inspected for the percentage of positively immunolabeled cells over the total number of cells in each region. First, an average value per section was calculated and then, a single value was determined by averaging the results of the three sections from each animal. 


\section{Neurobehavioral Examination}

Motor function score (MFS) was determined using the motor function scale as described by Farooque et al., where each rat was observed for at least 1 minute as they moved freely on a horizontal plane (8). Hip, knee, and ankle joint movements were recorded.

Inclined plane score (IPS) was obtained using the Rivlin and Tator's angle board test (21). The highest angle at which each rat could maintain its position for 5 seconds on an inclined plane was recorded weekly for each rat.

Both MFS and IPS were determined prior to the spinal cord injury and at the end of the 4th week following the spinal cord injury.

\section{Histopathological Examination}

After 4 weeks, the observed rats were sacrificed and spinal cord segments below the T9 level were removed under microscopy. These tissue samples were fixed in $10 \%$ formaldehyde and embedded in paraffin. Then, $5 \mu \mathrm{m}$ sections were cut from paraffin blocks and stained with hematoxylin and eosin. These slides were evaluated under light microscope to study histopathological changes (Figure 1A-D). Lesion size and spinal cord cross-sectional area at the lesion epicenter level were measured. The percentage of lesion size to the spinal cord area was calculated. Three sections with the largest lesion area were selected and the percentage of the lesion area was evaluated. A single average value per animal was obtained by averaging the measurements.

\section{Statistical Analysis}

Data were analyzed using SPSS 15.0 for Windows (SPSS Inc., Chicago, IL, USA). The results were expressed as mean \pm standard deviation (SD). Statistical analysis between



Figure 1: Representative microscopic images of spinal cord sections. A) Hematoxylin-eosin (H\&E) stained section taken 4 weeks after injury (400x). B-D) Immunohistochemal staining of cells by APAF-1, iNOS, and eNOS 24 hours after injury (400x). iNOS, eNOS, and APAF-1 immunoreactive cells are shown by arrows. iNOS: Inducible nitric oxide synthase; eNOS: endothelial nitric oxide synthase; APAF-1: apoptotic peptidase activating factor 1. 
groups was performed with the Kruskal-Wallis test. For dual comparison, the Mann-Whitney $U$ test was used. Differences were considered statistically significant at the $p<0.05$ level.

\section{RESULTS}

\section{Biochemical Examination}

MDA levels in the trauma, treatment, and vehicle groups were significantly higher compared to the control group (all $p<0.01$ ). The treatment group had significantly lower MDA levels than both the trauma and vehicle groups (all $p<0.01$ ).

Similarly, PC levels in the control group were significantly lower compared to all other groups (all $p<0.01$ ). Additionally, the treatment group had significantly reduced PC levels when compared to the trauma and vehicle groups, where the latter two did not differ significantly $(p<0.01, p<0.01$, and $p>0.05$, respectively).

rGSH levels were significantly decreased in the trauma, treatment, and vehicle groups when compared to those in the control group (all $p<0.01$ ). In the treatment group, rGSH levels were significantly higher than those in trauma and vehicle groups (all $p<0.01$ ).

Comparison of the biochemical parameters among groups is summarized in Table I.

\section{Immunohistochemical Examination}

iNOS, eNOS, and APAF-1 immunoreactivities significantly varied between the groups $(p<0.01)$. The treatment group had significantly lower iNOS and APAF-1 levels compared to the trauma and vehicle groups (all $\mathrm{p}<0.01$ ). eNOS was also significantly lower in the treatment group compared to the trauma and vehicle groups ( $p<0.05$ and $p<0.01$ respectively). iNOS, eNOS, and APAF-1 levels in the control group were significantly lower than those in the treatment group $(p<0.01$, $\mathrm{p}<0.05$ and $\mathrm{p}<0.01$ respectively) (Table II).

\section{Neurobehavioral Examination}

Pretrauma MFS and IPS scores did not differ among the groups ( $p>0.05)$. At the $4^{\text {th }}$ week, mean MFS scores were $6.0 \pm$ $0.0,2.2 \pm 1.3,4.2 \pm 0.8$, and $2.0 \pm 1.4$, while IPS scores were $58.8 \pm 3.11,28.8 \pm 6.3,48.2 \pm 1.9$, and $28.4 \pm 5.3$ for groups $1,2,3$ and 4 respectively.

The trauma, treatment, and vehicle groups had significantly lower MFS and IPS values compared to the control group (all $p<0.01)$. Animals in the treatment group scored significantly higher than those in trauma and vehicle groups in MFS (all $p<0.05)$. Similarly, treatment group scores for IPS were higher than those of the trauma and treatment groups (all $p<0.01$ ) (Figure 2).

\section{Histopathological Examination}

Histopathological changes such as necrosis, infiltration of inflammatory cells, and demyelination were present in all the groups except the control group (Figure 1A-D). The mean percentage of lesion area to spinal cord cross-sectional area was $34.7 \pm 3.55 \%, 24.3 \pm 2.59 \%$, and $36.0 \pm 2.77 \%$ in trauma, treatment, and vehicle groups, respectively. The lesion area in the treatment group was significantly lower than those in trauma and vehicle groups (all $\mathrm{p}<0.01$ ) (Table II).

Table I: Malondialdehyde, Protein Carbonyl, and Reduced Glutathione Levels Among the Groups

\begin{tabular}{lccr}
\hline & $\begin{array}{c}\text { Malondialdehyde (nmol/g) } \\
\text { (mean } \pm \text { SD) }\end{array}$ & $\begin{array}{c}\text { Protein carbonyl } \\
\text { (nmol/g) (mean } \pm \text { SD) }\end{array}$ & $\begin{array}{c}\text { Reduced glutathione } \\
\text { (nmol/g) (mean } \pm \text { SD) }\end{array}$ \\
\hline Group 1 (control) & $87.0 \pm 5.05$ & $44.2 \pm 4.70$ & $11.7 \pm 1.16$ \\
\hline Group 2 (trauma) & $148.8 \pm 4.26$ & $65.7 \pm 8.76$ & $5.5 \pm 1.35$ \\
\hline Group 3 (treatment) & $126.5 \pm 6,00^{*}$ & $54.9 \pm 2.52^{*}$ & $8.5 \pm 0.63^{*}$ \\
\hline Group 4 (vehicle) & $149.3 \pm 12.51$ & $72.7 \pm 13.84$ & $5.6 \pm 1.06$ \\
\hline
\end{tabular}

SD: standard deviation. ${ }^{*} p<0.01$ compared to groups 2 and 4.

Table II: iNOS, eNOS, and APAF-1 Percentage and Lesion Areas in Spinal Cord Sections among the Groups

\begin{tabular}{lcccc}
\hline & $\begin{array}{c}\text { iNOS (\%) } \\
\text { (mean } \pm \text { SD) }\end{array}$ & $\begin{array}{c}\text { eNOS (\%) } \\
\text { (mean } \pm \text { SD) }\end{array}$ & $\begin{array}{c}\text { APAF-1 (\%) } \\
\text { (mean } \pm \text { SD) }\end{array}$ & $\begin{array}{c}\text { Lesion area (\%) } \\
\text { (mean } \pm \text { SD) }\end{array}$ \\
\hline Group 1 (control) & $21.9 \pm 2.38$ & $20.7 \pm 1.40$ & $15.9 \pm 1.69$ & $0.0 \pm 0.0$ \\
\hline Group 2 (trauma) & $44.4 \pm 3.37$ & $36.7 \pm 2.98$ & $28.1 \pm 1.96$ & $34.7 \pm 3.55$ \\
\hline Group 3 (treatment) & $32.1 \pm 2.80^{*}$ & $29.9 \pm 1.32 \#$ & $22.1 \pm 1.89^{*}$ & $24.3 \pm 2.59^{*}$ \\
\hline Group 4 (vehicle) & $45.1 \pm 3.94$ & $36.5 \pm 3.08$ & $29.1 \pm 1.50$ & $36.0 \pm 2.77$ \\
\hline
\end{tabular}

APAF-1: Apoptotic peptidase activating factor 1, eNOS: Endothelial nitric oxide synthase, iNOS: Inducible nitric oxide synthase, SD: standard deviation. * $p<0.01$ compared to groups 2 and $4 ; \# p<0.05$ compared to group $2, p<0.01$ compared to group 4. 


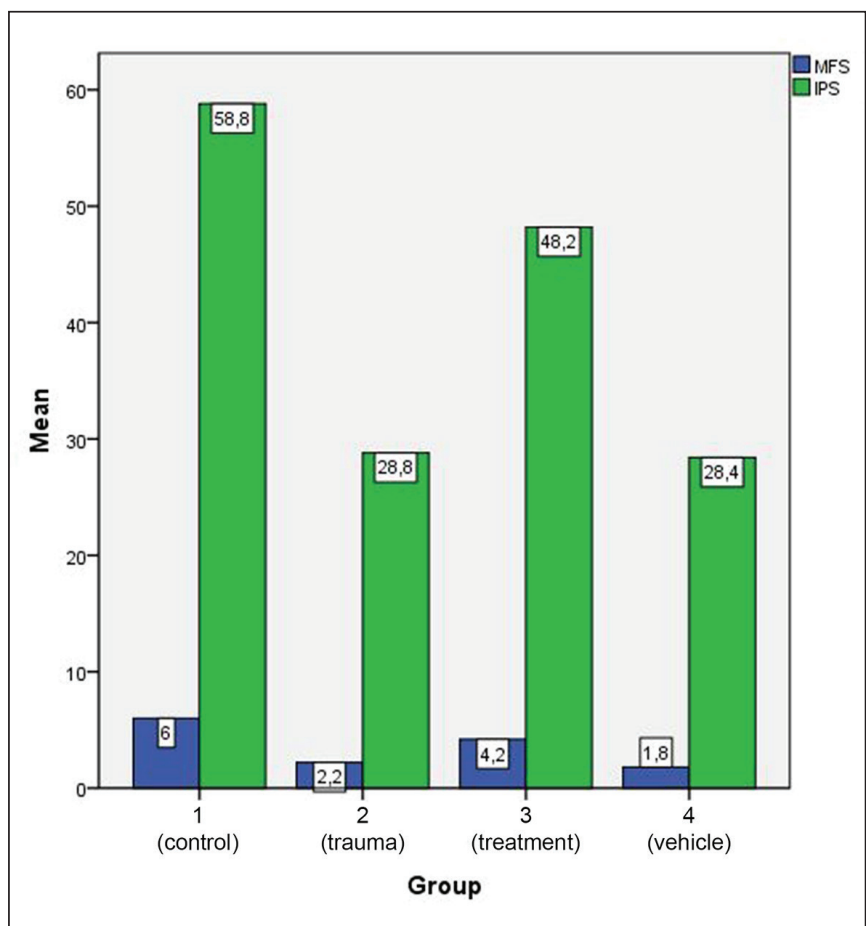

Figure 2: Mean motor function scores (MFS) and inclined plane scores (IPS) 4 weeks after the spinal cord injury.

\section{DISCUSSION}

In spinal cord injury, the primary injury results from acute compression or laceration of the spinal cord, which is impossible to treat. On the other hand, secondary injury begins with petechial hemorrhages in the gray matter and edema in the white matter, followed by myelin sheath destruction, axonal degeneration, and ischemic endothelial injury as early as in the $4^{\text {th }}$ hour. This leads to progressive axonal changes and the development of necrotic zones in the next few days (22). Macrophages, major inflammatory cells in $\mathrm{SCl}$, secrete high levels of proinflammatory cytokines such as tumor necrosis factor a (TNF-a), which is a potent activator of neutrophils, another major cell in secondary injury, as well as reactive nitrogen and oxygen intermediates, especially during the initial stages (14). Furthermore, accumulated macrophages secrete NG2 which inhibits neuronal regeneration (11). This cascade of events leads to necrosis and apoptosis of neurons, destruction of the axonal structure and myelin sheaths, which eventually result in functional loss. In an experimental SCI study, partial functional recovery was established by depletion of the macrophages (20).

Our study demonstrated that azathioprine, a DNA synthesis inhibitor, which is used primarily for immunosuppression, protects neural tissues in $\mathrm{SCl}$ when delivered within 30 minutes after an injury as shown by biochemical, histological, and functional measures. To the best of our knowledge, this is the second study demonstrating the neuroprotective effects of azathioprine in $\mathrm{SCl}$ and first in a traumatic $\mathrm{SCl}$ setting. The previous report studied the effect of azathioprine on spinal cord ischemia-reperfusion injury. The major difference from our study was the administration of azathioprine prior to the experimental ischemic injury (1). Although this practice may be feasible in aortic surgery where the spinal cord is under risk of ischemia, it is not possible to administer a drug in daily life prior to an injury. Our study showed that early administration of azathioprine following spinal cord trauma provides protection against oxidative damage as shown by reduced lipid peroxidation, protein carbonylation, and increased rGSH levels. Oxidative stress is one of the leading factors in secondary spinal cord injury $(2,10)$. Previous studies focusing on the prevention of the oxidative stress with various agents resulted in improved recovery $(2,3)$. Azathioprine's protective effect against oxidative stress might be directly related to its antioxidant properties, which are yet to be determined or indirectly, due to their diminishing effect on macrophages at the injury site, known to contribute to the production and mediation of free oxygen radicals (10).

Excessive nitric oxide levels, which is a result of upregulation of nitric oxide synthase (NOS) subtypes in $\mathrm{SCl}$, is also known to be neurotoxic (6). Macrophages are known to produce iNOS which have apoptotic and inflammatory properties, whereas azathioprine was shown to inhibit iNOS synthesis in macrophages (18). In our study, iNOS levels were increased at the injury site when no treatment was given, but were found to be diminished in those treated with azathioprine. It has been already demonstrated that azathioprine decreases iNOS synthesis in molecular studies, but to the best of our knowledge, it is the first time this is being demonstrated in an animal model. Also, to a lesser extent, it is possible that azathioprine's effect on macrophage migration at the injury site might have also played a role in low iNOS levels. Similarly, APAF-1 was found to be lower in the treatment group compared to the trauma and vehicle groups. This finding is consistent with the low iNOS levels and supports the protective effects of azathioprine against apoptosis.

Contusion in spinal the cord damages several ascending and descending tracks at the same time without any specific pattern. Therefore, biochemical or pathological measures remain insufficient to assess functional recovery. Behavioral tests are useful in these situations, where no specific injury pattern is present (20). Neurobehavioral evaluation of rats demonstrated functional recovery, supporting the biochemical, immunohistochemical, and pathological findings of this study.

We believe the neuroprotective effects of azathioprine is mostly determined by its effects on macrophages. However, we did not perform macrophage count on the samples in our study, which can be regarded as a limitation. Yet, several studies already demonstrated azathioprine's inhibitory effect on macrophage proliferation, activation, and migration $(9,17,19)$. On the other hand, these studies on the roles of macrophages in spinal cord injury focused mainly on the histopathological aspects, while ignoring the biochemical and immunohistochemical aspects $(1,4,14,20)$.

Methylprednisolone, though some opposing studies exist, has been shown to improve the functional outcome after $\mathrm{SCl}$ in animals by reducing the secondary damage that is attributed to reduced inflammation, lipid peroxidation, and apoptosis. 
However, high doses of methylprednisolone are needed to achieve the desired effects, which may lead to increased susceptibility to infections (16). Azathioprine is also widely used as an immunosuppressive and anti-inflammatory agent in a variety of diseases, although high concentrations are not needed to achieve therapeutic doses in SCI.

\section{CONCLUSION}

As a consequence, our study demonstrated the protective effects of azathioprine in $\mathrm{SCl}$ from the biochemical, histopathological, immunohistochemical, and behavioral aspects. It should be noted that further experimental studies are needed to investigate its short- and long-term effects depending on the dosage and optimal dosing schedule should be established prior to clinical studies.

\section{ACKNOWLEDGEMENTS}

Preparation for publication of this article is partly supported by Turkish Neurosurgical Society

\section{- REFERENCES}

1. Akgun S, Tekeli A, Kurtkaya O, Civelek A, Isbir SC, Ak K, Arsan S, Sav A: Neuroprotective effects of FK-506, L-carnitine and azathioprine on spinal cord ischemia-reperfusion injury. Eur J Cardiothorac Surg 25:105-110, 2004

2. Azbill RD, Mu X, Bruce-Keller AJ, Mattson MP, Springer JE: Impaired mitochondrial function, oxidative stress and altered antioxidant enzyme activities following traumatic spinal cord injury. Brain Res 765:283-290, 1997

3. Bilginer B, Onal MB, Narin F, Ustun H, Kilinc K, Akalan N: Antiapoptotic and neuroprotective effects of mycophenolate mofetil after acute spinal cord injury in young rats. Childs Nerv Syst 25:1555-1561, 2009

4. Blight AR: Delayed demyelination and macrophage invasion: A candidate for secondary cell damage in spinal cord injury. Cent Nerv Syst Trauma 2:299-315, 1985

5. Caspritz G, Hadden J: The Immunopharmacology of Immunotoxicology, and Immunorestoration. Toxicol Pathol 15: 320-332, 1987

6. Conti A, Miscusi M, Cardali S, Germano A, Suzuki H, Cuzzocrea S, Tomasello F: Nitric oxide in the injured spinal cord: Synthases cross-talk, oxidative stress and inflammation. Brain Res Rev 54:205-218, 2007

7. Ellman GL: Tissue sulfhydryl groups. Arch Biochem Biophys 82:70-77, 1959

8. Farooque M, Isaksson J, Jackson DM, Olsson Y: Clomethiazole (ZENDRA, CMZ) improves hind limb motor function and reduces neuronal damage after severe spinal cord injury in rat. Acta Neuropathol 98:22-30, 1999

9. Gassmann AE, Fruth Rv: The effect of azathioprine (Imuran) on the kinetics of monocytes and macrophages during the normal steady state and an acute inflammatory reaction. Blood 46:51-64, 1975

10. Jia Z, Zhu H, Li J, Wang X, Misra H, Li Y: Oxidative stress in spinal cord injury and antioxidant-based intervention. Spinal Cord 50:264-274, 2012
11. Jones LL, Yamaguchi Y, Stallcup WB, Tuszynski MH: NG2 is a major chondroitin sulfate proteoglycan produced after spinal cord injury and is expressed by macrophages and oligodendrocyte progenitors. J Neurosci 22:2792-2803, 2002

12. Koozekanani SH, Vise M, Hashemi RM, McGhee RB: Possible mechanisms for observed pathophysiological variability in experimental spinal cord injury by the method of Allen. $J$ Neurosurg 44:429-434, 1976

13. Lee YL, Shih K, Bao P, Ghirnikar RS, Eng LF: Cytokine chemokine expression in contused rat spinal cord. Neurochem Int 36:417-425, 2000

14. Leskovar A, Moriarty LJ, Turek JJ, Schoenlein IA, Borgens RB: The macrophage in acute neural injury: Changes in cell numbers over time and levels of cytokine production in mammalian central and peripheral nervous systems. J Exp Biol 203:1783-1795, 2000

15. Levine RL, Garland D, Oliver CN, Amici A, Climent I, Lenz A-G, Ahn BW, Ahaltiel S, Stadtman ER: Determination of carbonyl content in oxidatively modified proteins. Methods Enzymol 186:464-478, 1990

16. Mabon PJ, Weaver LC, Dekaban GA: Inhibition of monocyte/ macrophage migration to a spinal cord injury site by an antibody to the integrin aD: A potential new anti-inflammatory treatment. Exp Neurol 166:52-64, 2000

17. Marinković G, Hamers AAJ, de Vries CJM, de Waard V: 6-Mercaptopurine reduces macrophage activation and gut epithelium proliferation through inhibition of GTPase Rac1. Inflamm Bowel Dis 20:1487-1495, 2014

18. Moeslinger T, Friedl R, Spieckermann PG: Inhibition of inducible nitric oxide synthesis by azathioprine in a macrophage cell line. Life Sci 79:374-381, 2006

19. Pols TWH, Bonta PI, Pires NMM, Otermin I, Vos M, Vries MRd, Eijk Mv, Roelofsen J, Havekes LM, Quax PHA, Kuilenburg ABPv, Waard Vd, Pannekoek $\mathrm{H}$, Vries CJMd: 6-Mercaptopurine inhibits atherosclerosis in apolipoprotein $\mathrm{E}^{\star} 3$-leiden transgenic mice through atheroprotective actions on monocytes and macrophages. Arterioscler Thromb Vasc Biol 30:1591-1597, 2010

20. Popovich PG, Guan Z, Wei P, Huitinga I, Rooijen Nv, Stokes BT: Depletion of hematogenous macrophages promotes partial hindlimb recovery and neuroanatomical repair after experimental spinal cord injury. Exp Neurol 158:351-365, 1999

21. Rivlin AS, Tator $\mathrm{CH}$ : Objective clinical assessment of motor function after experimental spinal cord injury in the rat. $J$ Neurosurg 47:577-581, 1977

22. Taoka Y, Okajima K: Spinal cord injury in the rat. Prog Neurobiol 56:341-358, 1998

23. Tator $\mathrm{CH}$, Fehlings MG: Review of the secondary injury theory of acute spinal cord trauma with emphasis on vascular mechanisms. J Neurosurg 75:15-26, 1991

24. Uchiyama M, Mihara M: Determination of malonaldehyde precursor in tissues by thiobarbituric acid test. Anal Biochem 86:271-278, 1978 